\title{
Responses to Continuous Glucose Monitoring in Subjects with Type 1 Diabetes Using Continuous Subcutaneous Insulin Infusion or Multiple Daily Injections
}

\author{
David Rodbard, M.D.,', Lois Jovanovic, M.D., ${ }^{2}$ and Satish K. Garg, M.D. ${ }^{3}$
}

\begin{abstract}
Objective: We compared changes in response to unmasking of continuous glucose monitoring (CGM) in subjects with type 1 diabetes who use multiple daily injections (MDI) or continuous subcutaneous insulin infusion (CSII). Research Design and Methods: Use of real-time CGM (DexCom [San Diego, CA] SEVEN ${ }^{\circledR}$ ) was studied in 38 subjects using CSII and 26 using MDI. CGM output was masked during Week 1 and unmasked during Weeks 2 and 3. We evaluated changes in 16 criteria for quality of glycemic control and eight criteria for glycemic variability.

Results: All 24 criteria showed highly statistically significant improvement when considered simultaneously $(P<0.000001)$. For subjects using CSII, 18 of 24 criteria improved significantly (nominal $P<0.05$ ); for subjects using MDI, 16 of 24 criteria improved significantly $(P<0.05)$. Twelve of the comparisons remained significant $(P<0.05)$ after applying the overconservative Bonferroni correction for multiple comparisons. The percentage of glucose values within the range $80-140 \mathrm{mg} / \mathrm{dL}$ increased by $19 \%$ and $17 \%$ relative to their control values (Week 1) for subjects using MDI and CSII, respectively. Mean glucose, overall SD (SD ${ }_{T}$ ), SD between daily means $\left(\mathrm{SD}_{\boldsymbol{d m}}\right.$ ), mean amplitude of glycemic excursion (MAGE), and mean of daily differences (MODD) improved significantly. Responses to CGM display were not significantly different between the MDI and CSII subject groups for any of the 24 criteria considered individually or in groups of eight, 16, or 24 .
\end{abstract}

Conclusion: CGM has similar effectiveness in subjects with type 1 diabetes using either CSII or MDI.

\section{Introduction}

$\mathbf{U}$ SE OF CONTINUOUs glucose monitoring (CGM) has been demonstrated to improve glucose control in a wide range of patient populations. ${ }^{1-11}$ However, there remains a common misconception that CGM is primarily indicated for subjects who are using continuous subcutaneous insulin infusion (CSII) and that it might not be indicated in subjects who are using multiple daily injections of insulin (MDI). The effect of CGM on glycemic variability per se has been less studied $^{9-11}$ despite the numerous clinical and preclinical studies suggesting a linkage between glycemic variability and development of diabetes complications. ${ }^{12-17}$

Garg and Jovanovic ${ }^{2}$ demonstrated the beneficial effects of CGM in 85 patients with type 1 and type 2 diabetes using either CGM or MDI. We have recently reanalyzed the subset of data from 64 of those subjects with type 1 diabetes and 17 subjects with type 2 diabetes, using 20 criteria for quality of glycemic control and 28 criteria for glycemic variability, in- cluding several new criteria. ${ }^{9}$ We found highly statistically significant benefits of CGM, with many criteria showing statistical significance conservatively at the level of $P<0.0001$. The present study was undertaken to examine the question whether CGM with real-time display had similar effectiveness in subjects with type 1 diabetes who use CSII (insulin pump) and those who use MDI. We reanalyzed the data ${ }^{2}$ using newly developed and improved methodologies involving multiple criteria for quality of glycemic control and glycemic variability.

Portions of this study were presented as a late breaking poster at the $69^{\text {th }}$ Annual Scientific Sessions of the American Diabetes Association, New Orleans, LA, June 5-9, 2009. ${ }^{18}$

\section{Methods}

The characteristics of the patient population and the experimental design have been described previously. ${ }^{2}$ The study involved 64 subjects with type 1 diabetes studied at five

\footnotetext{
${ }^{1}$ Biomedical Informatics Consultants, LLC, Potomac, Maryland.

${ }^{2}$ Sansum Diabetes Research Institute, Santa Barbara, California.

${ }^{3}$ Barbara Davis Center for Diabetes, University of Colorado Denver, Aurora, Colorado.
} 
clinical centers within the United States. Subjects younger than 18 years of age and pregnant or lactating women were not eligible. Institutional Review Board approval and informed consent were obtained in all cases. Subjects were provided with a DexCom (San Diego, CA) SEVEN ${ }^{\circledR}$ sensor, transmitter, and receiver for a 3-week period. Display of the glucose sensor results was masked during Week 1 but made available ad libitum on demand during Weeks 2 and 3. Sensors were changed weekly. The subjects were presented with a brief review of the principles of diabetes self-management immediately prior to commencing the study. Subjects were allowed to use the CGM data as they thought appropriate; no specific algorithm or instructions were provided. No other changes were made in patient management.

\section{Subject characteristics}

Table 1 summarizes characteristics of the study population. ${ }^{2}$ For subjects with type 1 diabetes mellitus, there were no statistically significant differences between the MDI and CSII groups with respect to any of these eight characteristics using an unpaired two-sided Student's $t$ test $(P>0.05)$. The study population was $58.7 \%$ women and $89.5 \%$ Caucasian, $10 \%$ Hispanic. Among subjects using MDI, 52.0\% were female, $77.7 \%$ were Caucasian, and $7.4 \%$ were Hispanic. Among subjects using CSII, $61.5 \%$ were female, $100 \%$ were Caucasian, and $11.6 \%$ were Hispanic.

\section{CGM}

Subjects used a sensor for three consecutive 1-week periods as described previously. ${ }^{2}$ Sensor readings were masked dur- ing Week 1 but displayed during Weeks 2 and 3. Data were downloaded from the sensor devices on a weekly basis. We compared the properties of the glucose time series for Week 1 (CGM sensor masked) and Week 3 (CGM sensor unmasked) within subjects.

\section{Criteria for quality of glycemic control and glycemic variability}

We computed 16 criteria for quality of glucose control ${ }^{19-26}$ and 8 criteria for glycemic variability (Table 2), including several new methods. ${ }^{22,23}$ The number of criteria was reduced from 49 to 24 to reduce the problems associated with multiple comparisons, using the most sensitive criteria as identified in our previous studies, ${ }^{9}$ so as to reduce redundancy, and avoid use of criteria that are relatively difficult to interpret.

Measures of quality of glucose control. These included the mean of all glucose values (all days, all times of day), designated Mean $\boldsymbol{T}$, percentage of glucose values within three specified target ranges $(80-200 \mathrm{mg} / \mathrm{dL}, 80-140 \mathrm{mg} / \mathrm{dL}, 70$ $180 \mathrm{mg} / \mathrm{dL}$ ), percentage in the hypoglycemic range (below 50 or $80 \mathrm{mg} / \mathrm{dL}$ ), percentage in the hyperglycemic range (above 180 or $250 \mathrm{mg} / \mathrm{dL}),{ }^{2}$ Schlichtkrull's $M_{\mathbf{1 0 0}},{ }^{19}$ Wojcicki's $J$ index, ${ }^{20}$ Kovatchev's Low Blood Glucose Index (LBGI) and High Blood Glucose Index (HBGI), ${ }^{21}$ Rodbard's Index of Glycemic Control (IGC), ${ }^{22,23}$ and Hill's GRADE score. ${ }^{24}$ (The adjustable parameters for the IGC were set as follows: a target range of $80-140 \mathrm{mg} / \mathrm{dL}$ [LLTR $=80, \mathbf{U L T R}=140$ ]; exponent for excursions above ULTR $a=1.1$; exponent for excursions below LLTR $b=2.0$; linear scaling factors: $c=d=30 .^{22,23}$ )

Table 1. Characteristics of 81 Study Subjects with Type 1 Diabetes Mellitus Using CSII or MDI

\begin{tabular}{|c|c|c|c|c|c|}
\hline & Mean & $S D$ & SEM & Minimum & Maximum \\
\hline \multicolumn{6}{|c|}{ Age (years) } \\
\hline CSII & 42.9 & 13.4 & 2.1 & 20.2 & 68.4 \\
\hline MDI & 36.9 & 12.7 & 2.5 & 19.8 & 70.0 \\
\hline \multicolumn{6}{|c|}{ Duration of diabetes (years) } \\
\hline CSII & 21.9 & 13.2 & 2.1 & 4.0 & 49.0 \\
\hline MDI & 17.0 & 11.4 & 2.3 & 4.0 & 50.0 \\
\hline \multicolumn{6}{|c|}{ Baseline A1C (\%) } \\
\hline CSII & 7.3 & 0.77 & 0.1 & 5.8 & 9.2 \\
\hline MDI & 7.9 & 1.33 & 0.3 & 5.9 & 11.5 \\
\hline \multirow{2}{*}{\multicolumn{6}{|c|}{$\begin{array}{l}\text { Mean CGM glucose } \\
\text { during Week } 1(\mathrm{mg} / \mathrm{dL})\end{array}$}} \\
\hline & & & & & \\
\hline CSII & 169.0 & 31.1 & 5.0 & 114.1 & 248.5 \\
\hline MDI & 182.1 & 31.8 & 6.2 & 112.8 & 238.3 \\
\hline \multicolumn{6}{|c|}{ Height $(\mathrm{cm})$} \\
\hline CSII & 169.7 & 9.8 & 1.6 & 137.2 & 189.8 \\
\hline MDI & 171.8 & 12.6 & 2.5 & 147.3 & 196.2 \\
\hline \multicolumn{6}{|c|}{ Weight (kg) } \\
\hline CSII & 75.8 & 16.3 & 2.7 & 54.5 & 124.4 \\
\hline MDI & 82.3 & 21.8 & 4.4 & 54.9 & 159.0 \\
\hline \multicolumn{6}{|c|}{$\operatorname{BMI}\left(\mathrm{kg} / \mathrm{m}^{2}\right)$} \\
\hline CSII & 26.2 & 4.3 & 0.7 & 19.3 & 36.4 \\
\hline MDI & 27.8 & 5.9 & 1.2 & 20.1 & 41.5 \\
\hline \multicolumn{6}{|c|}{ SMBG frequency (per day) } \\
\hline CSII & 5.9 & 1.9 & 0.3 & 3.0 & 10.0 \\
\hline MDI & 5.5 & 2.4 & 0.5 & 2.0 & 10.0 \\
\hline
\end{tabular}

There were no statistically significant differences between the two groups of subjects in any of these eight parameters as evaluated by an unpaired $t$ test $(P>0.05$, difference not significant). BMI, body mass index; ISF, interstitial fluid; SMBG, self-monitoring of blood glucose. 


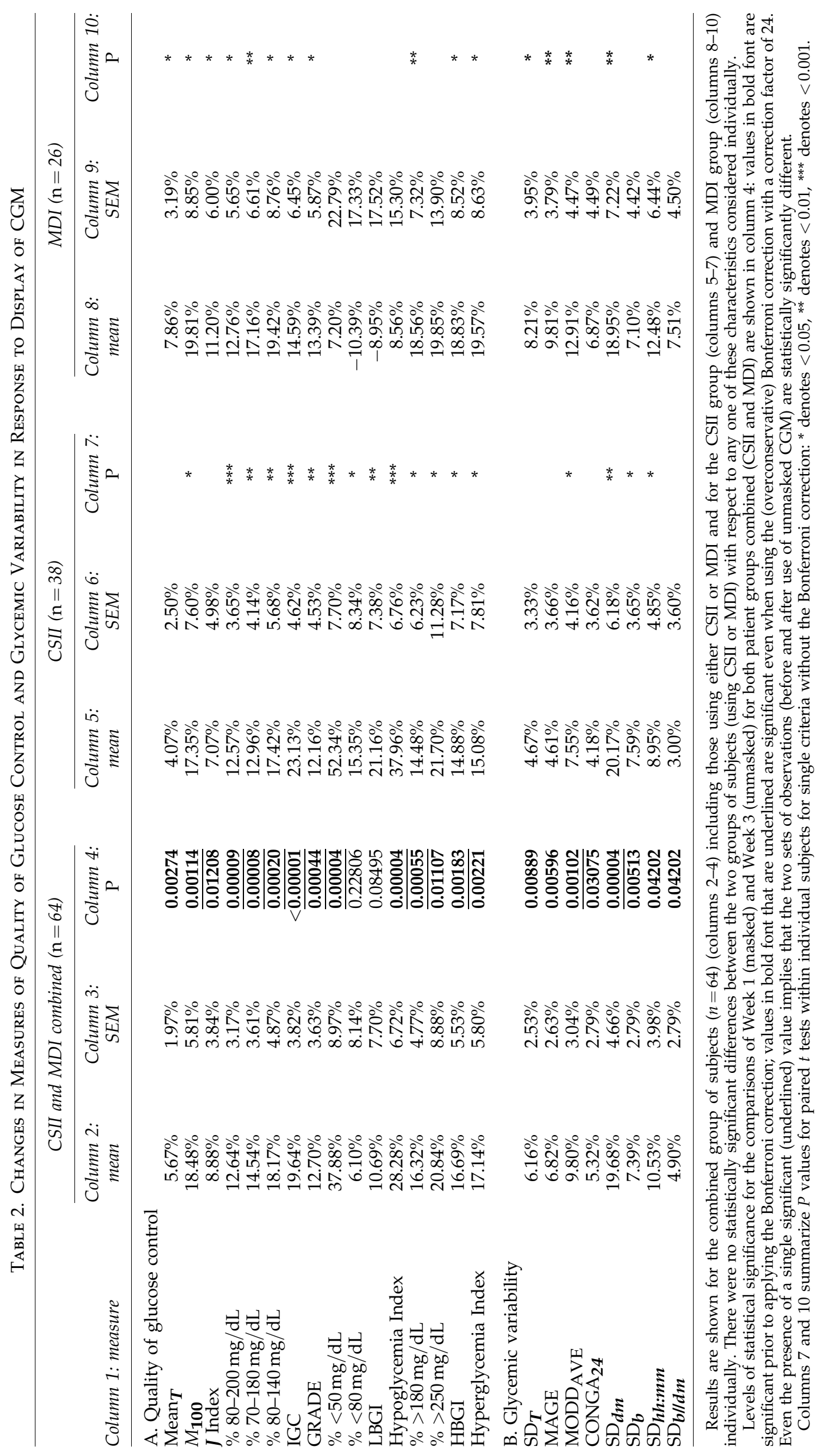


Measures of glycemic variability. These included three "classical" measures: mean amplitude of glycemic excursion (MAGE) ${ }^{26}$ mean of daily differences (MODD) ${ }^{26}$ and the overall or "total" SD $\left(\mathrm{SD}_{\mathrm{T}}\right) .{ }^{9,22,23}$ MODD was originally defined only for a 2 -day series, ${ }^{26}$ so it must be generalized to apply to a multiday series. $\mathrm{MODD}_{A V E}$ is the average of MODD obtained by the original method of calculation for all consecutive pairs of days for any subject. ${ }^{22,23}$ We also examined several new types of SD values, including the SD of daily means $\left(\mathrm{SD}_{d m}\right)$, the $\mathrm{SD}$ between days-within time points $\left(\mathrm{SD}_{\boldsymbol{b}}\right)$, the $\mathrm{SD}$ between days-within time points after correction for changes in daily means $\left(\mathrm{SD}_{\boldsymbol{b}} / / \boldsymbol{d} \boldsymbol{m}\right)$, the SD between time points $\left(\mathrm{SD}_{\boldsymbol{h h}: \mathbf{m m})}\right)$, and the $\mathrm{SD}$ within days $\left(\mathrm{SD}_{\boldsymbol{w}}\right){ }^{21,22}$ We also examined $\mathrm{CONGA}_{24} \cdot{ }^{25}$ Computations were performed using SAS (Cary, NC) software and using a dedicated software program for the calculation of MAGE. An Excel (Microsoft, Redmond, WA) spreadsheet was also developed for computation of the several types of SD $\left(\mathrm{SD}_{\boldsymbol{T}}, \mathrm{SD}_{\boldsymbol{d}}, \mathrm{SD}_{\boldsymbol{b}}\right.$, $\mathrm{SD}_{\boldsymbol{b} / / \boldsymbol{d} \boldsymbol{m}}, \mathrm{SD}_{\boldsymbol{w}}, \mathrm{SD}_{\boldsymbol{h} \boldsymbol{h}: \boldsymbol{m m}}$ ) (cf. the supplementary online appendix to Rodbard ${ }^{23}$ ). Interrelationships among these measures are discussed elsewhere. ${ }^{22,23}$

\section{Hypothesis testing}

We first examined the effects of CGM on the entire group of 64 subjects with type 1 diabetes, pooling the data from subjects using CSII and MDI. Comparisons between time periods (Week 1 vs. Week 3 ) were made using paired $t$ tests so that each subject could be used as his or her own control. The mean relative percentage improvement in all 24 criteria, in the 16 criteria for quality of glycemic control, and in the eight criteria for glycemic variability were tested versus the null hypothesis using a one-sided $t$ test for the CSII and MDI groups separately. Improvement corresponded to an increase in the percentage of glucose values within a specified target range (80-200, 70-180, or $80-140 \mathrm{mg} / \mathrm{dL}$ ) or a decrease in any of the other 21 criteria. The percentage improvement in the mean value of each criterion during Week 3 (within subjects) was calculated as the mean change in each parameter expressed as a percentage of the mean value during the control period (Week 1).

We then evaluated the CSII and MDI groups separately and evaluated the difference in magnitude of responses to CGM using two-sided unpaired $t$ tests. The mean percentage improvement for the 24,16 , or eight criteria in the CSII and MDI groups was compared using a two-sided $t$ test. After demonstrating that the CSII and MDI groups combined showed a statistically significant change in response to use of CGM, we then tested each of the 24 criteria individually in both groups of subjects. A one-sided $t$ test was used when the direction of the change could be predicted a priori. Results are presented as $P$ values before application of a Bonferroni correction to compensate for the use of multiple comparisons. In view of the high degree of correlation of the parameters (cf. the supplementary online appendix for Rodbard et al. ${ }^{9}$ ), the Bonferroni correction is extremely overconservative.

We examined the relationships of changes in criteria between Week 1 and Week 3 to the baseline hemoglobin A1c (A1C). We performed tests to evaluate whether the regression lines for the MDI and CSII groups were compatible by: (a) fitting least-squares regression lines to the two groups separately (involving four parameters, i.e., two slopes and two intercepts); (b) fitting another regression line after pooling the data from the two groups, involving two parameters, i.e., one slope and one intercept; and (c) comparing the residual sum of squares using an $F$ test based on the extra-sum of squares principle.

\section{Results}

Table 2 shows comparisons of Week 3 (CGM values unmasked) with Week 1 (masked), for subjects using CSII and for subjects using MDI. When all subjects with are considered simultaneously (combining the MDI and CSII groups), 22 of the 24 criteria were significantly improved after unmasking of the real time display of CGM data, including 14 of 16 criteria for quality of glycemic control and all eight criteria for glycemic variability. When the 38 subjects using CSII are considered, 18 of 24 criteria showed statistically significant improvement; when the 26 subjects using MDI are considered, 16 of 24 criteria improved significantly $(P<0.05)$ between Week 1 and Week 3. There were no statistically significant differences in the percentage improvement observed between Week 1 (masked) and Week 3 (unmasked) for any of the 24 criteria between the MDI and CSII groups, whether one considers each criterion individually, the mean percentage improvement for all 24 criteria, the 16 criteria for quality of glycemic control, or the eight criteria for glycemic variability.

Figure 1 displays the percentage improvement in glucose values in Week 3 relative to Week 1 for 16 criteria-eight reflecting quality of glycemic control and eight reflecting glycemic variability-using each subject as his or her own control, i.e., using a paired analysis within subjects.

We examined the relationships between the changes in response to unmasking of CGM measured for each subject and the subject's baseline A1C level. There was a statistically significant linear relationship for several parameters. Figure 2 shows a representative example for one criterion, the GRADE score. The magnitude of the benefit derived from unmasking of real-time display of CGM appeared to be linearly related to the baseline A1C. There were no statistically significant differences in the regression lines for the CSII and MDI patient groups. Similar results were observed for all criteria examined (see Supplementary Appendix at www.liebertonline.com/ dia). Subjects with elevated baseline A1C values showed the greatest benefit in measures of hyperglycemia, euglycemia, and overall quality of glycemic control. In contrast, for subjects whose baseline A1Cs were close to the target levels of $6.5-7 \%$ at baseline, unmasking of CGM for the brief time frame of only 2 weeks resulted in little or no benefit in terms of measures of hyperglycemia and euglycemia. However, this group (with A1C 6.5-7\%) showed the greatest improvement in four measures of hypoglycemia (percentage of glucose values below $80 \mathrm{mg} / \mathrm{dL}$, percentage of glucose values below $50 \mathrm{mg} / \mathrm{dL}, \mathrm{LBGI}$, and Hypoglycemia Index).

Numerous criteria for evaluation of glycemic control and glycemic variability have been proposed previously. It is not evident a priori which measure or measures are most important and clinically relevant. Accordingly, we have examined the majority of the available measures to identify the ones that are most sensitive to the effect of this particular therapeutic intervention. 

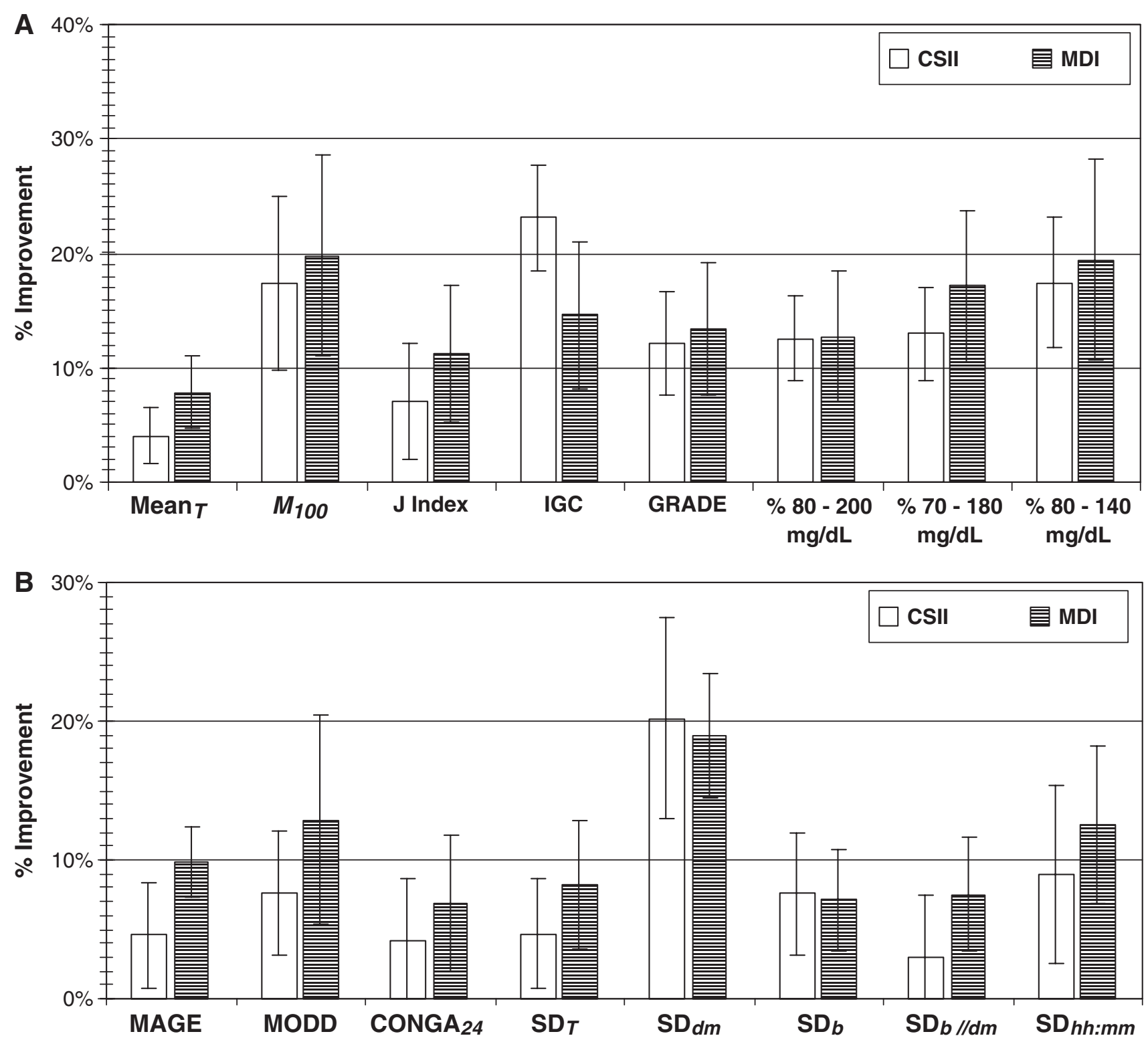

FIG. 1. Responses to display of CGM ( \pm 1 SEM) expressed as percentage improvement between Week 1 and Week 3 for measures of (A) quality of glycemic control and (B) glycemic variability. Responses for subjects using CSII are shown in open columns;

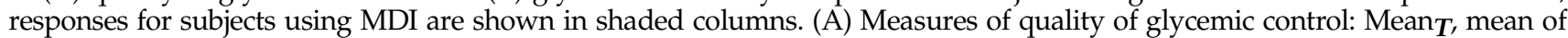
glucose for all days and all times of day; $M_{\mathbf{1 0 0}}$, Schlichtkrull's $M$ with a reference value of $100 \mathrm{mg} / \mathrm{dL}$; Index; IGC (Rodbard's Index of Glycemic Control ${ }^{2,23}$ ); GRADE (GRADE score of Hill et al. ${ }^{24}$ ); and percentage of glucose values within three specified

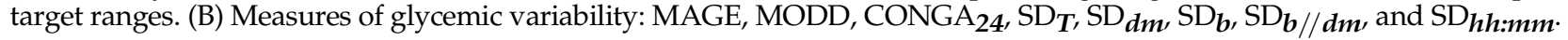

\section{Measures of quality of glucose control}

We first tested whether there was an effect of CGM when the CSII and MDI groups are combined for each of the individual criteria. A significant effect was seen in 14 of 16 criteria at the nominal $P$ value of $P<0.05$ (Table $2 \mathrm{~A}$, column 4 ). We applied an extremely conservative Bonferroni correction for multiple comparisons, using a factor of 24-the total number of criteria examined in the present study, such that a $P$ value of $0.05 / 24=0.0021$ is required to achieve statistical significance. Ten of the 16 comparisons remained significant and are shown in bold underlined font in column 4 of Table $2 \mathrm{~A} . M_{\mathbf{1 0 0}}$,
J, HBGI, LBGI, IGC, and GRADE improved for the combined group of subjects (CSII and MDI).

Having established the presence of a significant change in response to CGM for the entire group, we then analyzed the CSII and MDI groups separately (Table 2A, columns 5-7 and 8-10, respectively). A similar effect was seen in both the CSII and MDI groups of subjects, with 14 and 11 of the 24 criteria significant at the nominal $P<0.05$ level without use of the Bonferroni correction. Because of the smaller sample sizes (38 and 26 for the CSII and MDI groups, respectively), the effect of CGM was not statistically significant within either of these subgroups after applying the Bonferroni correction. The 


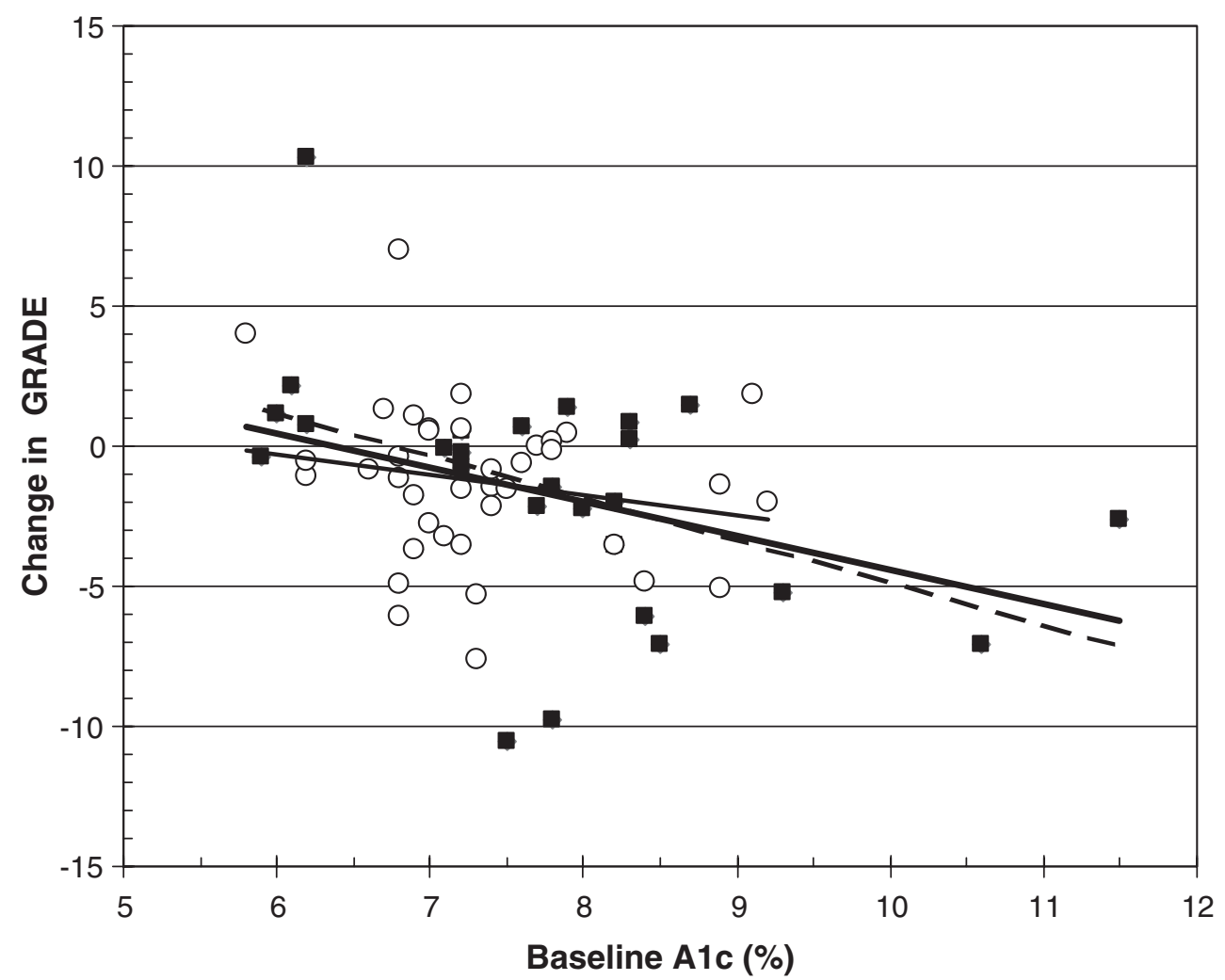

FIG. 2. Relationship of change in one criterion of overall quality of glycemic control, GRADE, versus baseline A1C. Regression lines are shown for the groups of subjects using MDI (solid squares, dashed line), CSII (open circles, thin line), and for both groups combined (heavy line).

percentage of glucose values in the target ranges 80-200, 70-180, and 80-140 $\mathrm{mg} / \mathrm{dL}$ improved for subjects using either CSII or MDI. The mean glucose $\left(\right.$ Mean $\left._{T}\right)$ improved significantly $(P<0.05)$ for the MDI group but not in the CSII group. Three criteria for hypoglycemia (LBGI, Hypoglycemia Index, and percentage $<50 \mathrm{mg} / \mathrm{dL}$ ) improved significantly in the CSII group but not in the MDI group. CGM resulted in an increase in glucose values in the range $50-80 \mathrm{mg} / \mathrm{dL}$ but a decrease in percentage of values below $50 \mathrm{mg} / \mathrm{dL}$ in the MDI group, corresponding to a reduction in severe hypoglycemia but an increase in moderate degrees of hypoglycemia. This was reflected in discordance in the responses for LBGI and Hypoglycemia Index, which are highly correlated with percentage of glucose values below $80 \mathrm{mg} / \mathrm{dL}$ and $50 \mathrm{mg} / \mathrm{dL}$, respectively. The results for IGC also reflect the changes in the Hypoglycemia Index. There is an excellent correlation $(r=+0.88)$ between the percentage improvement in 12 criteria for the CSII and MDI groups, when the four criteria for hypoglycemia are excluded (cf. Figure A17 of the Supplementary Appendix at www.liebertonline.com/dia).

\section{Measures of glycemic variability}

When one combines results from subjects using CSII and MDI, then all eight criteria for glycemic variability are improved at the nominal $P<0.05$ level (Table 2B, column 4). After application of the Bonferroni correction using a factor of 24, two criteria, $\mathrm{MODD}_{\boldsymbol{A} \boldsymbol{V} E}$ and $\mathrm{SD}_{\boldsymbol{d} \boldsymbol{m}}$, remain significant at the $P<0.05$ level, indicating a reduction in day-to-day vari- ability. If we examine the mean relative percentage improvement in all eight criteria considered simultaneously, we find a statistically significant response $(P<0.01)$ in the subjects using either CSII or MDI (cf. Table A2 of Supplementary Appendix at www.liebertonline.com/dia). A $t$ test comparing the CSII and MDI groups did not show any significant difference between groups. Further, there was a very high degree of correlation of the percentage improvement in each of the eight criteria for the CSII and MDI groups. The SD $d m$ decreased significantly in both groups. The total variability, as reflected in $\mathrm{SD}_{\boldsymbol{T}}$ and MAGE, was significantly improved for MDI group but not in the CSII group. The between daywithin time point variability $\left(\mathrm{SD}_{\boldsymbol{b}}\right)$ improved by a nearly identical $7.59 \%$ and $7.10 \%$ for the CSII and MDI groups, respectively. However, partly due to the different number of subjects in the two groups, the change in the CSII group was significant at the $P<0.05$ level, whereas the change in $\mathrm{SD}_{\boldsymbol{b}}$ for the MDI group was not.

\section{Discussion}

\section{Selection of criteria for responses to CGM}

We utilize 16 measures of quality of glycemic control. Four of these are related to hyperglycemia (percentage of glucose values $>250 \mathrm{mg} / \mathrm{dL}$, percentage $>180 \mathrm{mg} / \mathrm{dL}$, HBGI, and Hyperglycemic Index). Four criteria are related to hypoglycemia (percentage of glucose values $<50 \mathrm{mg} / \mathrm{dL}$, percentage $<80 \mathrm{mg} / \mathrm{dL}$, LBGI, and Hypoglycemia Index). Three are related to "euglycemia" (percentage of glucose values within 
80-200, 70-180, and 80-140 mg/dL). Five criteria are "general" indices, including Mean Glucose $\left(M_{T}\right), M_{\mathbf{1 0 0}}, J$, IGC, and GRADE. We utilized eight measures of glycemic variability, including measures of total variability $\left(\mathrm{SD}_{T}\right)$, within-day variability (MAGE, SDhh:mm), and between-day variability $\left(\mathrm{MODD}_{A \boldsymbol{}}, \mathrm{SD}_{\boldsymbol{d} \boldsymbol{m}}, \mathrm{SD}_{\boldsymbol{b}}, \mathrm{SD}_{\boldsymbol{b}} / / \boldsymbol{d} \boldsymbol{m}, \mathrm{CONGA}_{24}\right)$. The definitions, properties, and interrelationships among these measures are described elsewhere (Rodbard et al. ${ }^{9}$ and Rodbard ${ }^{23}$ ). We also examined the responses to six additional criteria, which gave similar results.

\section{Effectiveness of CGM in type 1 diabetes}

Several previous studies have reported the effectiveness of CGM in subjects with type 1 diabetes using either CSII or MDI. ${ }^{1-11,18,27}$ Nevertheless, there is a popular misconception among many clinicians that CGM is indicated primarily for subjects using CSII or for subjects already in relatively good to excellent glycemic control. As a result, some health insurance companies have refused to provide reimbursement for CGM in subjects using MDI and for those subjects with relatively poor glycemic control. The present study was underpowered to detect effects in only the MDI or CSII subgroups of subjects. However, when we combine results from both groups, and when we combine results from the multiple (24) criteria, then the benefit of introducing unmasked CGM-without any other specific instructions to the patient as to how best to use the information-is highly statistically significant at the $P<0.000001$ level. By use of a single comparison, we avoid the problem of multiple comparisons. The effects of CGM are evident in the majority of criteria when examined individually. Even after applying the excessively conservative Bonferroni correction, we find that several comparisons are significant at the $P<0.05$ level. Thus, the effect of CGM can be clearly and unambiguously demonstrated in both the CSII and MDI groups. The effect of CGM is also evident when we examine individual criteria (Table 2) at the usual or nominal $P<0.05$ level. However, when examining these criteria individually, the results would not be significant after applying the Bonferroni correction. (The 24 criteria are highly correlated so that one is not making 24 independent comparisons. Accordingly, the Bonferroni correction is overconservative. If all of the criteria were perfectly correlated, then the Bonferroni correction would not or should not be used-all criteria would be giving the same result. In the present data set, several of the criteria show correlations of $0.8-0.9$ (cf. online supplementary materials associated with reference 9 ).

\section{Similarity of response in subjects using CSII versus MDI}

The present study (Table 2, Figs. 1 and 2, and Supplementary Appendix at www.liebertonline.com/dia) demonstrates that there are similar and statistically indistinguishable responses to CGM in groups of patients using CSII or MDI. This is true whether we examine each of the criteria individually or in groups of eight, 16, or 24 (see Supplementary Appendix at www.liebertonline.com/dia). The power to detect a difference increases as we use a composite response variable such as mean percentage improvement. The responses for subjects using CSII and MDI are very highly correlated for all eight measures of glycemic variability and for 12 of the 16 measures of quality of glycemic control. Only the four measures of hypoglycemia display discordance between the results for patients using CSII and MDI. This finding deserves further examination in independent, longer-term studies and may depend on subject selection criteria and the set of instructions provided to the subjects regarding the recommended methods to respond to the information provided by CGM.

\section{Relationship of response to baseline $A 1 C$}

The recently reported Juvenile Diabetes Research Foundation study indicates the benefit of CGM in subjects with type 1 diabetes who are well controlled (A1C $<7.0 \%) .{ }^{10}$ That study involved 62 patients using CSII and CGM, but only five of these were using MDI, so an analysis by treatment modality is not possible. Similarly, Danne et al. ${ }^{11}$ have recently reported beneficial effects of CGM in 48 patients with type 1 diabetes, of whom 39 were using CSII and nine were using MDI. For this subject population, Danne et al. ${ }^{11}$ reported no relationship of improvements in A1C or glucose SD with initial A1C when using a protocol involving 20 days with CGM masked followed by 40 days with CGM unmasked (see Fig. 1 of Danne et al. $\left.{ }^{11}\right)$. Weinzimer et al. ${ }^{8}$ have also reported that CGM is effective in subjects using MDI.

In the present study, the availability of real-time display of CGM was equally efficacious in groups being treated with CSII or with MDI (Table 2, Fig. 1). The relationships of responses to baseline A1C were also indistinguishable in subjects using CSII or MDI (Fig. 2, and Supplementary Appendix at www.liebertonline.com/dia).

\section{Experimental design}

The present study uses the data as reported previously by Garg and Jovanovic. ${ }^{2}$ Patients with type 1 diabetes were selected on the basis of their willingness to utilize CGM for a period of 3 weeks. They were not randomized to a treatment group of CSII or MDI. Hence, there is the possibility that the two groups might differ in terms of various aspects of their diabetes. We examined several characteristics of the two patient groups (Table 1) and did not find any statistically significant differences. However, these subjects might differ in their level of experience with self-management, motivation, education in terms of diabetes management, and ability and willingness to adjust insulin dosages. Although these differences did not reach statistical significance, subjects who use CSII tended to be older, have a longer duration of diabetes, a lower baseline A1C, and use self-monitoring of blood glucose more frequently than the subjects using MDI (Table 1). These and related factors may contribute to the subtle differences between groups, e.g., as related to frequency and severity of hypoglycemia. Accordingly, it would be desirable to repeat the present study, using randomization to assign subjects to CSII or MDI groups. Such an experimental design would entail many practical difficulties, as one would need to recruit subjects who were willing and able to use both CSII and MDI, preferably using a crossover experimental design.

\section{Statistical methods}

We have used a variety of statistical methods to examine a modest-sized dataset. We first confirmed the effect of CGM in the subset of subjects with type 1 diabetes using either CSII or MDI. (Our previous analyses of these data examined these 
same subjects combined with 17 subjects with type 2 diabetes. ${ }^{9,27}$ ) We were able to confirm the effect of CGM using individual criteria (Table 2) and using the Bonferroni correction. We then examined the effects of CGM separately for the CSII and MDI groups, using individual criteria (Table 2). Finally, we used the composite variables (percentage improvement) for eight, 16, or 24 criteria for CSII and MDI separately and compared the responses for the two groups using an unpaired $t$ test (Tables A1, A2, and A3 of supplementary Appendix at www.libertonline.com/dia). The results obtained were remarkably consistent irrespective of the statistical methods used.

The present study provides new insights regarding the clinical utility of CGM with real-time display of glucose levels in subjects with diabetes and provides insights into the performance of both "classical" and recently described criteria for quality of glucose control and glycemic variability, in terms of their relative sensitivity for detection of changes in response to a therapeutic intervention, i.e., unmasking of CGM. These insights should be helpful in the design and analysis of future studies.

\section{Conclusions}

Subjects with type 1 diabetes showed significant improvement in 16 measures of quality of glycemic control and eight measures of glycemic variability in response to unmasking of real-time displays of CGM data irrespective of whether they were using CSII or MDI. There were no significant differences between the responses in the CSII and MDI groups for any of the 24 criteria examined. The magnitude of improvement was generally greater in subjects with a higher baseline $\mathrm{A} 1 \mathrm{C}$ in this short-term study, with identical relationships for the CSII and MDI groups of subjects.

\section{Acknowledgments}

Bradley Matsubara, M.D. played an important role in the initiation of this study and provided many helpful comments. Katherine Nakamura, Ph.D. performed extensive statistical analyses of the data. This study was supported by DexCom, Inc., San Diego, CA. Dr. Matsubara and Dr. Nakamura are employees of DexCom, Inc.

\section{Author Disclosure Statement}

The Barbara Davis Center for Diabetes, Aurora CO, and the Sansum Diabetes Research Institute, Santa Barbara CA, received grants or contracts from DexCom, Inc. for support of clinical research studies which generated the data reported here. D.R. has served as a consultant to DexCom, Inc., and to the Barbara Davis Center for Diabetes.

\section{References}

1. Garg S, Zisser H, Schwartz S, Bailey T, Kaplan R, Ellis S, Jovanovic L: Improvement in glycemic excursions with a transcutaneous, real-time continuous glucose sensor: a randomized controlled trial. Diabetes Care 2006;29:44-50.

2. Garg S, Jovanovic L: Relationship of fasting and hourly blood glucose levels to $\mathrm{HbA1C}$ values: safety, accuracy, and improvements in glucose profiles obtained using a 7-day continuous glucose sensor. Diabetes Care 2006;29:2644-2649.
3. Bailey TS, Zisser HC, Garg SK: Reduction in hemoglobin A1C with real-time continuous glucose monitoring: results from a 12-week observational study. Diabetes Technol Ther 2007;9:203-210.

4. Garg SK, Kelly WC, Voelmle MK, Ritchie PJ, Gottlieb PA, McFann KK, Ellis SL: Continuous home monitoring of glucose: improved glycemic control with real-life use of continuous glucose sensors in adult subjects with type 1 diabetes. Diabetes Care 2007;30:3023-3025.

5. Juvenile Diabetes Research Foundation Continuous Glucose Monitoring Study Group, Tamborlane WV, Beck RW, Bode BW, Buckingham B, Chase HP, Clemons R, Fiallo-Scharer R, Fox LA, Gilliam LK, Hirsch IB, Huang ES, Kollman C, Kowalski AJ, Laffel L, Lawrence JM, Lee J, Mauras N, O'Grady M, Ruedy KJ, Tansey M, Tsalikian E, Weinzimer S, Wilson DM, Wolpert H, Wysocki T, Xing D: Continuous glucose monitoring and intensive treatment of type 1 diabetes. $\mathrm{N}$ Engl J Med 2008;359:1464-1476.

6. Deiss D, Bolinder J, Riveline JP, Battelino T, Bosi E, TubianaRufi N, Kerr D, Phillip M: Improved glycemic control in poorly controlled patients with type 1 diabetes using realtime continuous glucose monitoring. Diabetes Care 2006;29:2730-2732.

7. Mastrototaro J, Shin J, Marcus A, Sulur G; STAR 1 Clinical Trial Investigators: The accuracy and efficacy of real-time continuous glucose monitoring sensor in patients with type 1 diabetes. Diabetes Technol Ther 2008;10:385-390.

8. Weinzimer S, Xing D, Tansey M, Fiallo-Scharer R, Mauras N, Wysocki T, Beck R, Tamborlane W, Ruedy K; Diabetes Research in Children Network (DirecNet) Study Group: FreeStyle Navigator continuous glucose monitoring system use in children with type 1 diabetes using glargine-based multiple daily dose regimens: results of a pilot trial Diabetes Research in Children Network (DirecNet) Study Group. Diabetes Care 2008;31:525-527.

9. Rodbard D, Bailey T, Jovanovic L, Zisser H, Kaplan R, Garg $\mathrm{S}$ : Improved quality of glycemic control and reduced glycemic variability with use of continuous glucose monitoring. Diabetes Technol Ther 2009;11:717-723.

10. Juvenile Diabetes Research Foundation Continuous Glucose Monitoring Study Group: The effect of continuous glucose monitoring in well-controlled type 1 diabetes. Diabetes Care 2009;32:1378-1383.

11. Danne T, de Valk HW, Kracht T, Walte K, Geldmacher R, Soelter L, von dem Berge W, Welsh ZK, Bugler JR, Lange K, Kordonouri $\mathrm{O}$ : Reducing glycaemic variation in type 1 diabetes self-management with a continuous glucose monitoring system based on wired enzyme technology. Diabetologia 2009;52:1496-1503.

12. Hirsch IB, Brownlee M: The effect of glucose variability on the risk of microvascular complications in type 1 diabetes. Diabetes Care 2007;30:186-187.

13. Monnier L, Mas E, Ginet C, Michel F, Villon L, Cristol JP, Colette C: Activation of oxidative stress by acute glucose fluctuations compared with sustained chronic hyperglycemia in patients with type 2 diabetes. JAMA 2006;295:16811687.

14. Wentholt IM, Kulik W, Michels RP, Hoekstra JB, DeVries JH: Glucose fluctuations and activation of oxidative stress in patients with type 1 diabetes. Diabetologia 2008;51:183190.

15. Ceriello A, Esposito K, Piconi L, Ihnat MA, Thorpe JE, Testa $\mathrm{R}$, Boemi M, Giugliano D: Oscillating glucose is more deleterious to endothelial function and oxidative stress than 
mean glucose in normal and type 2 diabetic patients. Diabetes 2008;57:1349-1354.

16. Diabetes Research in Children Network (DirecNet) Study Group: The effect of glucose variability on the risk of microvascular complications in type 1 diabetes. Diabetes Care 2007;30:185.

17. Kilpatrick ES, Rigby AS, Atkin SL: The effect of glucose variability on the risk of microvascular complications in type 1 diabetes. Diabetes Care 2006;29:1486-1490.

18. Rodbard D, Jovanovic L, Garg S: Impact of continuous glucose monitoring (CGM) on glycemic variability and control in patients with type 1 diabetes using multiple daily injections (MDI) vs insulin pump [late breaking abstract LB2]. http://professional.diabetes.org/Abstracts_Display.aspx? TYP $=1 \& C I D=74882$ (accessed September 29, 2009).

19. Schlichtkrull J, Munck O, Jersild M: The M-valve, an index of blood-sugar control in diabetics. Acta Med Scand 1965; 177:95-102.

20. Wójcicki JM: "J"-index. A new proposition of the assessment of current glucose control in diabetic patients. Horm Metab Res 1995;27:41-42.

21. Clarke W, Kovatchev BP: Statistical tools to analyze continuous glucose monitor data. Diabetes Technol Ther 2009;11(Suppl 1):S-45-S-54.

22. Rodbard D: Interpretation of continuous glucose monitoring data: glycemic variability and quality of glycemic control. Diabetes Technol Ther 2009;11(Suppl 1):S55-S67.
23. Rodbard D: New and improved methods to characterize glycemic variability using continuous glucose monitoring. Diabetes Technol Ther 2009;11:551-565.

24. Hill NR, Hindmarsh PC, Stevens RJ, Stratton IM, Levy JC, Matthews DR: A method for assessing quality of control from glucose profiles. Diabet Med 2007;24:753-758.

25. McDonnell CM, Donath SM, Vidmar SI, Werther GA, Cameron FJ: A novel approach to continuous glucose analysis utilizing glycemic variation. Diabetes Technol Ther 2005;7: 253-263.

26. Service FJ, O’Brien PC, Rizza RA: Measurements of glucose control. Diabetes Care 1987;10:225-237.

27. Rodbard D, Jovanovic L, Garg S, Matsubara B, Nakamura K, Bailey T, Zisser H, Kaplan R: Reduced glycemic variability with the use of real-time continuous glucose monitoring [abstract 208-OR]. http://professional.diabetes.org/ Abstracts_Display.aspx?TYP $=1 \& C I D=74519$ (accessed August 31, 2009).

Address correspondence to: David Rodbard, M.D. Biomedical Informatics Consultants LLC 10113 Bentcross Drive Potomac, MD 20854-4721

E-mail: drodbard@comcast.net 
